\title{
Product of Perpendicular Diameters
}

National Cancer Institute

\section{Source}

National Cancer Institute. Product of Perpendicular Diameters. NCI Thesaurus. Code C135513.

The diameter multiplied by its perpendicular diameter. 